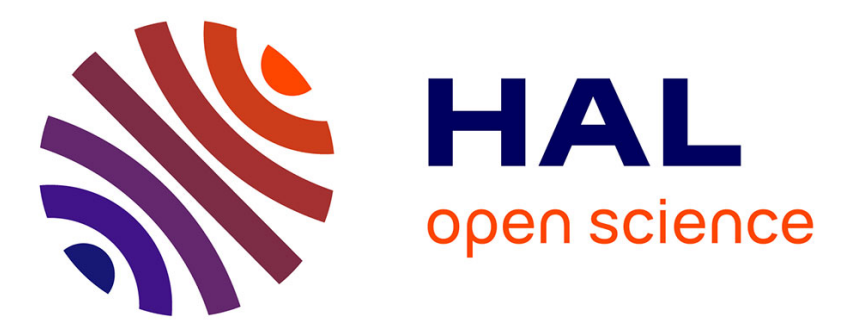

\title{
Conductance fluctuations in gold point contacts: an atomistic picture
}

Hubert Klein, Thomas Leoni, Philippe Dumas, Remi Zoubkoff, Andrés Saúl

\section{To cite this version:}

Hubert Klein, Thomas Leoni, Philippe Dumas, Remi Zoubkoff, Andrés Saúl. Conductance fluctuations in gold point contacts: an atomistic picture. Nanotechnology, 2012, 23, pp.235707. 10.1088/09574484/23/23/235707. hal-01075133

\section{HAL Id: hal-01075133 \\ https://hal.science/hal-01075133}

Submitted on 13 Sep 2017

HAL is a multi-disciplinary open access archive for the deposit and dissemination of scientific research documents, whether they are published or not. The documents may come from teaching and research institutions in France or abroad, or from public or private research centers.
L'archive ouverte pluridisciplinaire HAL, est destinée au dépôt et à la diffusion de documents scientifiques de niveau recherche, publiés ou non, émanant des établissements d'enseignement et de recherche français ou étrangers, des laboratoires publics ou privés. 


\title{
Conductance fluctuations in gold point contacts : an atomistic picture
}

\author{
Hubert Klein, ${ }^{*}$ Thomas Leoni, ${ }^{\dagger}$ Philippe Dumas, Remi Zoubkoff, and Andrés Saúl \\ CINaM UPR 3118 CNRS, Campus de Luminy, Case 913, 13288 Marseille cedex 9, France
}

(Dated: November 29, 2011)

\begin{abstract}
This paper concerns an experimental and theoretical study of the transition between two consecutive conductance plateaus as obtained in breaking gold contact experiments. The experimental measurements performed at $100 \mathrm{~K}$ with a scanning tunneling microscope and variable elongation speeds show that the transitions between consecutive plateaus can appear in the conductance traces as an abrupt conductance step, a smooth quasicontinuous change or as large amplitude conductance fluctuations. The theoretical calculations based on a non-orthogonal tight-binding Hamiltonian have shown that for a given deformation there are several structures having close and competing energies.

We discuss the relation between the temperature, sampling frequency, stretching speed and energy barriers which can explain the conditions for the observation of the three kinds of conductance traces.
\end{abstract}

\section{INTRODUCTION}

In breaking gold contact experiments using a scanning tunneling microscope (STM) or a mechanically controlable break junction (MCBJ), conductance plateaus are commonly observed for values close to multiples of the conductance quantum ${ }^{1,2} G_{0}=2 e^{2} / h$. These plateaus are usually separated by abrupt discontinuities. The former are related to elastic deformations of the system while the latter are the signature of plastic transformations or structural rearrangements. ${ }^{3}$ For given experimental conditions, if the overall behavior is the same, occurrences and extension of plateaus may vary from one experiment to another, which implies the use of statistical analysis.

Indeed, conductance histograms obtained from hundreds or thousands of elongation cycles were extensively used more than ten years ago to extract the signature of conductance quantization. $^{4-7}$ More recently, J. N. Armstrong et $a l .{ }^{8}$ have shown that the conductance histograms should be understood as a quasicontinuous distribution of conductance levels corresponding to a bunch of different atomic configurations. For a given elongation cycle, T. Leoni et al. ${ }^{9}$ have shown that successive conductance plateaus (corresponding to two and one $G_{0}$ ), are statistically independent events.

Time resolved experiments performed at low temperature $(4 \mathrm{~K})$ on gold contacts ${ }^{10}$ have shown that small amplitude conductance fluctuations (few percents of $G_{0}$ ) can occur during the plateaus. They have been explained as reversible transitions between two different atomic structures of the contact. Tsutsui et al. ${ }^{11}$ analyzed the dependence of the switching rate of the two-level fluctuations (of the order of $0.5 G_{0}$ ) with the applied bias to estimate the effective electronic temperature of a $\mathrm{Zn}$ atom-sized contact. Huntington et al. ${ }^{12}$ have performed a detailed experimental study of the dependence of the conductance traces under mechanical strain. By stretching a gold contact they have found that the transition between consecutive plateaus involves multilevel conductance fluctuations arising from discrete metastable atomic configurations.

This paper follows the paper of Huntington et al. ${ }^{12}$ and concerns an experimental and theoretical study of the transitions between two consecutive plateaus. In
Section II we present the experimental measurements of conductance-elongation curves of gold contacts at $100 \mathrm{~K}$ using a STM. We show that the same transition can appear in the conductance traces as an abrupt conductance step, a smooth quasicontinuous change or as large amplitude conductance fluctuations. In Section III we show, using theoretical calculations based on a non-orthogonal tight binding Hamiltonian, that these fluctuations correspond to transitions of a multi-level system. The transitions occur between local minima of the total energy of the system having different conductances. Finally in Section IV we discuss the relation between the experimental parameters (temperature, sampling frequency, stretching speed) and a theoretically calculated one (energy barrier) which can explain the conditions for the observation of the three kind of conductance traces.

\section{EXPERIMENTS}

\section{A. Methods}

We have performed breaking junction experiments using an ultra-high vacuum STM (VT-Omicron), where the sample is cooled down to $100 \mathrm{~K}$ using a liquid nitrogen cryostat. The temperature is recorded and regulated using a temperature controller (Lakeshore 330). We use a gold thin film evaporated on mica sheet ${ }^{13}$ as the first electrode and a $0.25 \mathrm{~mm}$ gold wire (99.99\%, Goodfellow) for the STM tip acting as the second one.

Conductance versus elongation traces (called $G(t)$ in the following, because the elongation is performed at constant speed and is thus proportional to time), are recorded using a dedicated software described in detail elsewhere. ${ }^{9,14}$ Briefly, while the STM regulation loop is maintained off, the tip is brought into contact with the surface prior to breaking off the junction by pulling the tip away with variable stretching speeds. Conductance data are simultaneously acquired at a sampling frequency of $10 \mathrm{kHz}$. The STM regulation loop is then activated and the cycle repeated automatically thousands of times. 


\section{B. Results}

Figure 1 shows several non consecutive $G(t)$ traces recorded at $100 \mathrm{~K}$ following the above mentioned procedure with a stretching speed of $100 \mathrm{~nm} / \mathrm{s}$.

One can clearly see small amplitude $0.1 G_{0}$ conductance spikes which are rarely observed at room temperature but have been reported previously in low temperature $4 \mathrm{~K}$ experiments. ${ }^{10}$ We will come back to this small amplitude fluctuations in Section IV.

We focus here on the transitions between two consecutive conductance plateaus. The traces in Fig. 1 show that these transitions can be abrupt or smooth. The abrupt

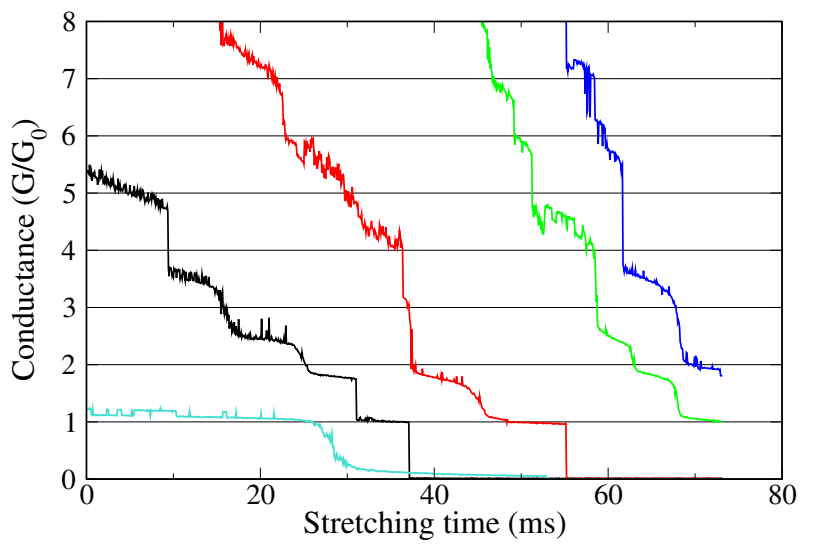

Figure 1. (color online) $\mathrm{G}(\mathrm{t})$ traces recorded at $\mathrm{T}=100 \mathrm{~K}$ and a stretching speed of $100 \mathrm{~nm} / \mathrm{s}$.

conductance steps, historically related to the atomic rearrangements of the junction during the breaking process, have been observed both at room and low temperature. The smooth transitions occurring between two conductance values separated by a $\Delta G$ of the order of $G_{0}$ last typically $10 \mathrm{~ms}$ in our experimental conditions, corresponding to an elongation of one nanometer.

In Fig. 2 we show several smooth transitions extracted from a set of data recorded at 100K. For the sake of clarity, these curves have been shifted with respect to the central point of the transition and are plotted versus an elongation axis. There is certain universality in these curves as can be seen from the low dispersion of the elongation range where the smooth transition occurs $\Delta z \simeq 1$ $\mathrm{nm}$.

We have also acquired $G(t)$ traces at a much lower stretching speed of $0.05 \mathrm{~nm} / \mathrm{s}$ as shown in Fig. 3. In addition to the small amplitude spikes (about $0.1 G_{0}$ ) already observed in the traces shown in Fig. 1, this $G(t)$ trace also exhibits conductance bistabilities of larger amplitude (about $0.6 G_{0}$ ). These large amplitude fluctuations appears in the form of random telegraphic noise.

Shot noise of an amplitude close to $G_{0}$ may correspond to the reversible opening of a conduction channel. Such a large bistability has been reported for a superconducting $\mathrm{Nb}$ contact. ${ }^{15}$ It was attributed to the formation of a

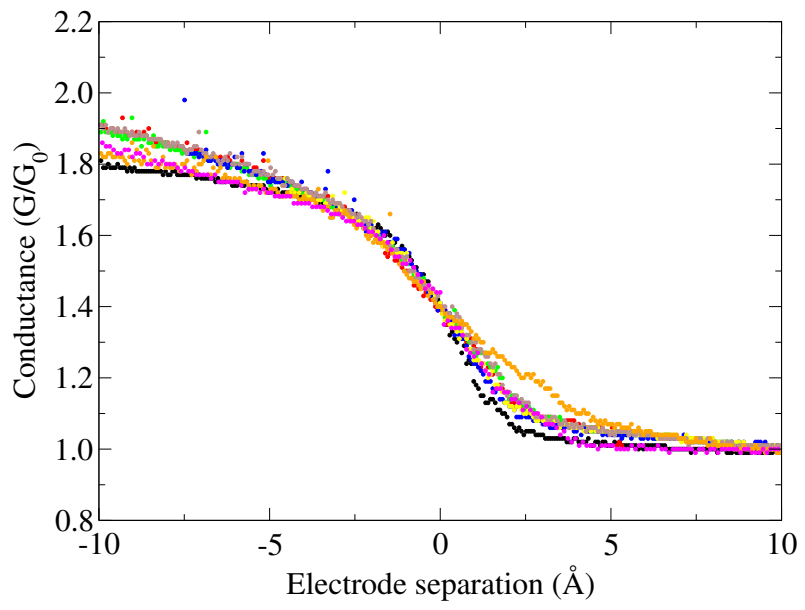

Figure 2. (color online) Conductance transition between 2 and $1 G_{0}$ extracted from several $G(t)$ traces recorded at 100 $K$. The traces have been centered on the electrode separation axis, with respect to the central point of the transition.

dimer which strongly modify the density of states in the superconducting gap, and thus altering the transmission of the conduction channels. As this mechanism does not stand for a gold contact, in our case, the reversible opening of a conduction channel can be due to the reversible fluctuation of the system between metastable states of the system having different conductances. ${ }^{12}$

If we interpret the large amplitude fluctuations presented in Fig. 3 as being those of a two level system, the average switching frequency is $15 \mathrm{~Hz}$ and the mean lifetimes in the high and low conductance states are in the range of 50-100 ms. The $1 / f^{2}$ dependency of the spectral density shown in Fig. 4, is an indication of a non-stationary random process. It is noteworthy that the statistics are not significant enough (195 of such transitions recorded) to prove undoubtedly the random nature of the process.

The only difference between the $G(t)$ traces of Figures 1 and 3 is the pulling speed of the electrodes, 100 and $0.05 \mathrm{~nm} / \mathrm{s}$ respectively. In both experiments, the duration of the transition corresponds to an elongation of the electrodes of the same order of magnitude, respectively 1 and $0.5 \mathrm{~nm}$ for Figures 1 and 3 .

In order to understand the interrelation between the role played by the different experimental parameters (elongation speed, temperature, sampling frequency) on the observation of the three classes of conductance traces, one needs to get insight on the atomistic mechanisms involved. We have thus undertaken computer simulations of the traction of gold nanowires.

\section{COMPUTER SIMULATIONS}

We have performed Molecular Dynamics (MD) simulations with an Environment Dependent Tight Binding 

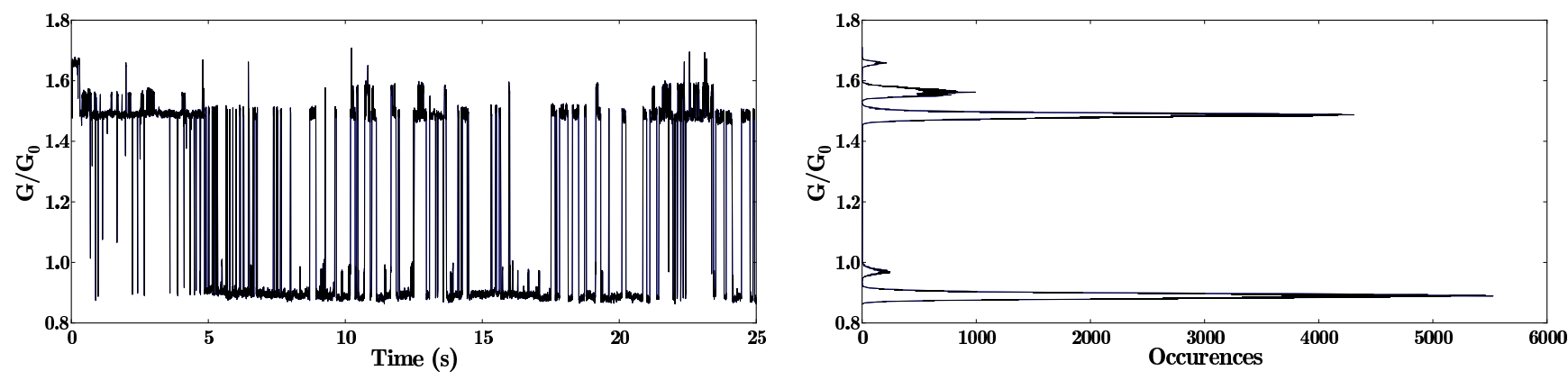

Figure 3. (color online) $\mathrm{G}(\mathrm{t})$ traces recorded at $\mathrm{T}=100 \mathrm{~K}$ and a stretching speed of $0.05 \mathrm{~nm} / \mathrm{s}$. Left : Conductance of a gold atomic wire versus time. The average switching frequency is $15 \mathrm{~Hz}$. Right : Histogram of the conductance trace shown in the left side.

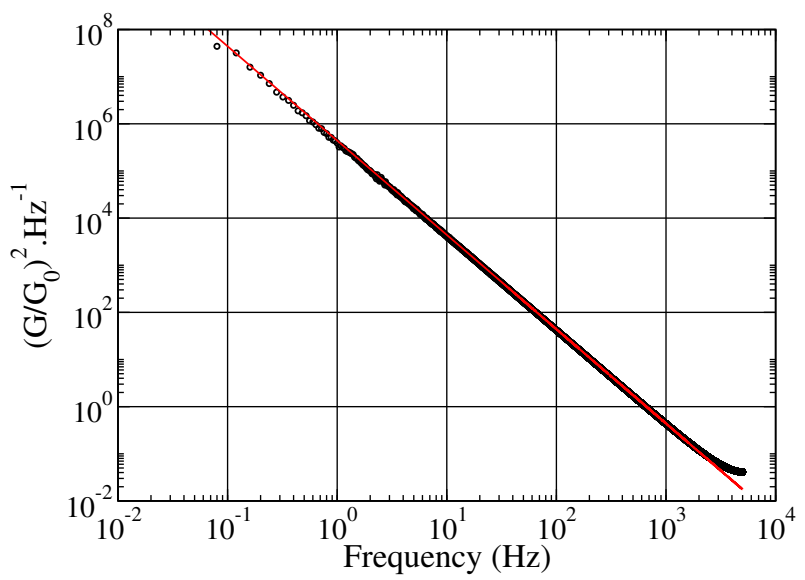

Figure 4. (color online) The black dots represent the power spectral density of the $G(t)$ trace of Fig. 3. The red line is a $1 / f^{2}$ fit which is an indication of a random non-stationary process.

Hamiltonian. ${ }^{16,17}$ The parameters of this Hamiltonian were obtained by fitting total energy differences and electronics properties to state of the art Density Functional calculations. The main advantage of this parametrized Tight Binding Hamiltonian is that the band energy (calculated as sum of eigenvalues) provides the full total energy without the requirement of additional pair potentials.

As the system under study presents atoms with different environments a self-consistent procedure for the charge transfer ${ }^{18}$ was employed. A Verlet algorithm ${ }^{19}$ was used to integrate the equations of motion with a time step of $1 \mathrm{fs}$. The temperature was controled using a Berendsen thermostat ${ }^{20}$ which has the effect to couple the system to an external bath. Traction was simulated by performing a succession of 2000 steps of molecular dynamics at $50 \mathrm{~K}$ followed of elongations of the periodic boundary condition along the nanowire axis of $0.1 \AA$.

The initial structure, oriented along the [110] direction contains 85 atoms and have a initial period of $14.25 \AA$. At the beginning of the traction process, the surface of the nanowire adopts a dense atomic structure with a (111) hexagonal local environment. Then, the surface curls to form a gold nanotube ${ }^{21,22}$ in order to avoid edges between the four $\{111\}$ planes that can be formed perpendicular to the nanowire axis. This part of the evolution process will be discussed elsewhere ${ }^{23}$, we focus here in the last stages before the formation of the monoatomic wire.
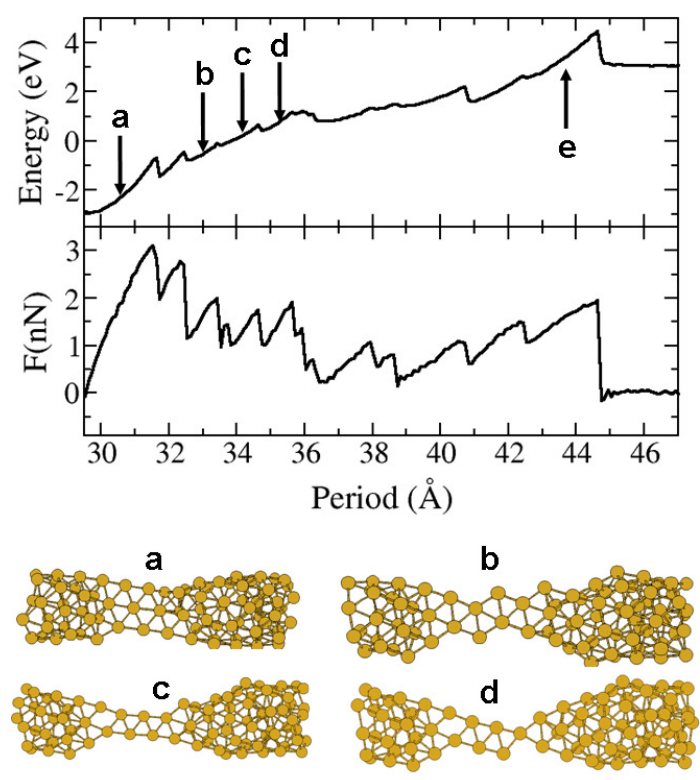

e

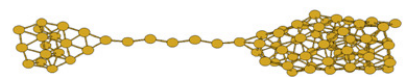

Figure 5. (color online) Energy and global force applied on the system as a function of the period during the deformation process. The reference of energy is arbitrary. The snapshots labeled a to e correspond to the configuration of the system for the periods marked by the arrows in the energy plot.

Figure 5 shows the total energy and global force of the system as a function of the period during the formation 
of the mono-atomic wire. In a first time a planar structure with hexagonal local environment (structure a) is formed. This structure evolves to form a biatomic chain (structures b and c) and the transition between biatomic (structure c) to monoatomic (structure d) takes place at a period of about $34.5 \AA$. From d to e, six atoms are included to form a seven atoms long chain. One can see a small but noticeable zig-zag as predicted by SanchezPortal et al. ${ }^{24}$ The six kinks between d to e in the evolution of the force are the signature of the structural changes related with the incorporation of the atoms in the chain. The linear evolution of the force between the kinks corresponds to elastic deformations.

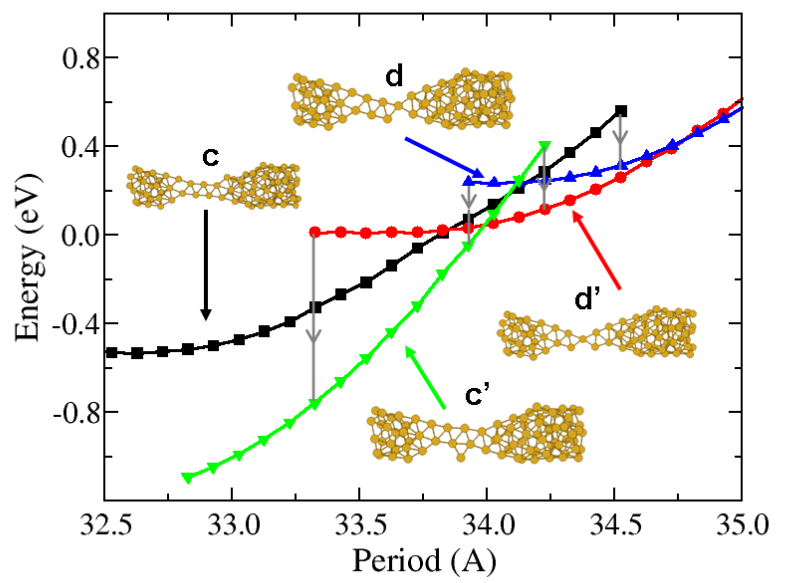

Figure 6. (color online) Energy as a function of the period calculated at $T=0$ for four different atomic configurations using a quenched Molecular Dynamics algorithm. The structures c and d are those obtained from the Molecular Dynamics simulations of the traction of the gold nanowires (Figure 5).

Assuming a simple model for the calculation of the conductance, the transition between two and one $G_{0}$ should correspond to the transition between two and one atomic section point contact (configurations $\mathrm{c}$ and $\mathrm{d}$ ).

In order to study in more detail this transition we have compressed and expanded the two configurations, i.e., beginning with the configuration just before (structure c) or just after (structure d) the transition, we have modified the period and minimized the energy using a quenched Molecular Dynamics algorithm ${ }^{25}$. The resulting energy versus deformation curves are shown in black for structure c and in grey (blue online) for structure d in Fig. 6. As can be seen, structure c can be elastically compressed but its period cannot be increased above $34.5 \AA$, it deforms plastically to a new single atom section structure labeled d'. At this period, this single atom configuration has a slightly lower energy than the structure d obtained during the Molecular Dynamics simulation. Structure d cannot be elastically compressed bellow $33.92 \AA$, it transforms plastically in another structure which present a two atoms contact labeled c'. It exists for periods between 32.82 and $34.22 \AA$. As expected the two single atom contact structures d and d' can be stabilized for longer periods, they both transform to structure c' when they are compressed.

We have not searched systematically all the possible metastable structures for every period, but it is clear that there are many others. For example, the adatom in configuration c' can turn around the biatomic wire giving rise to similar two atom contact structures. The picture that emerges from these calculations is that for every period there are several structures having close and competing energies. As shown by the parabolic shape of the energy curves these structures can be elastically deformed in a given range of period and they transform plastically to a different structure if stretched or compressed outside this range. For a given period the average time spent in every configuration is proportional to its Boltzmann weight and the transition probability to jump from one configuration to the other can be estimated from transition state theory from the corresponding energy barriers.

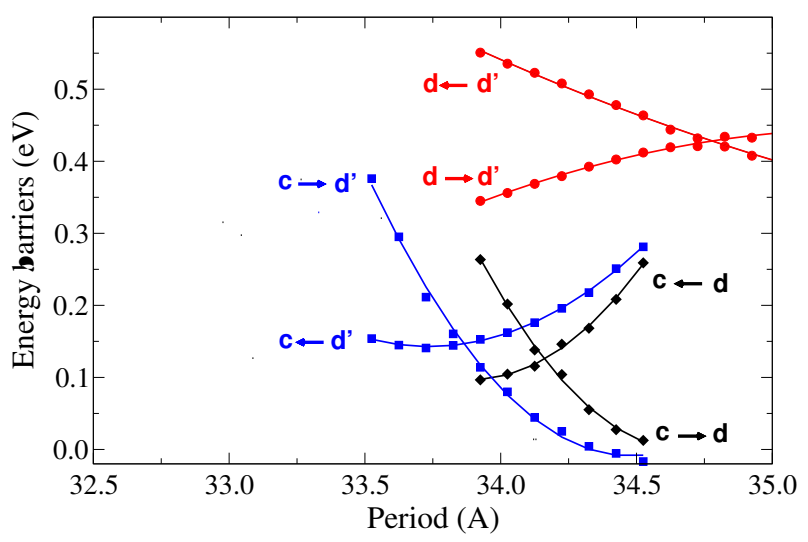

Figure 7. (color online) Energy barrier between some atomic configurations presented in Fig. 6 (circles : d and d', squares : $\mathrm{c}$ and d', and diamonds : c and d) as a function of the period.

Using structures c, d, and d' as initial and final states we have calculated the activation energies as a function of the deformation (see Fig. 7). The reaction path and the saddle point energy was determined using the nudged elastic band algorithm (NEB). ${ }^{26,27}$ We were unable to calculate transition to and from configuration c' using this algorithm. As can be seen the energy barriers vary with the deformation from 0 to $500 \mathrm{meV}$.

\section{DISCUSSION}

The above described computer simulations show that the system will perform transitions between local energy minima which can have different conductances. Due to the exponential dependency, the actual values of the energy barriers have a dramatic influence on the switching frequency. For example, at 100K, a decrease of the energy barrier from $0.22 \mathrm{eV}$ to $0.11 \mathrm{eV}$ increases the switching 
frequency of the $G(t)$ trace in Fig. 3 from $15 \mathrm{~Hz}$ to $8 \mathrm{MHz}$. Taking into account the experimental limit imposed by the instrument sampling time, it is clear that the observation of switching phenomena at a given temperature is only possible for a narrow distribution of energy barriers. To be more precise, let us define three characteristic times.

i) The switching time $t_{s w}$ between two metastable configurations. As it is the inverse of the switching frequency, it can be written from transition state theory as

$$
t_{s w} \simeq \frac{1}{\nu_{0}} \exp \left(\frac{E_{b}}{k T}\right)
$$

where $E_{b}$ is the energy barrier involved in this process, $k$ the Boltzmann constant, $T$ the temperature and $\nu_{0}=$ $510^{12} \mathrm{~Hz}$ an average phonon frequency. ${ }^{28}$ Fig. 8 shows the dependency of $t_{s w}$ with $T$ for different values of the energy barrier.

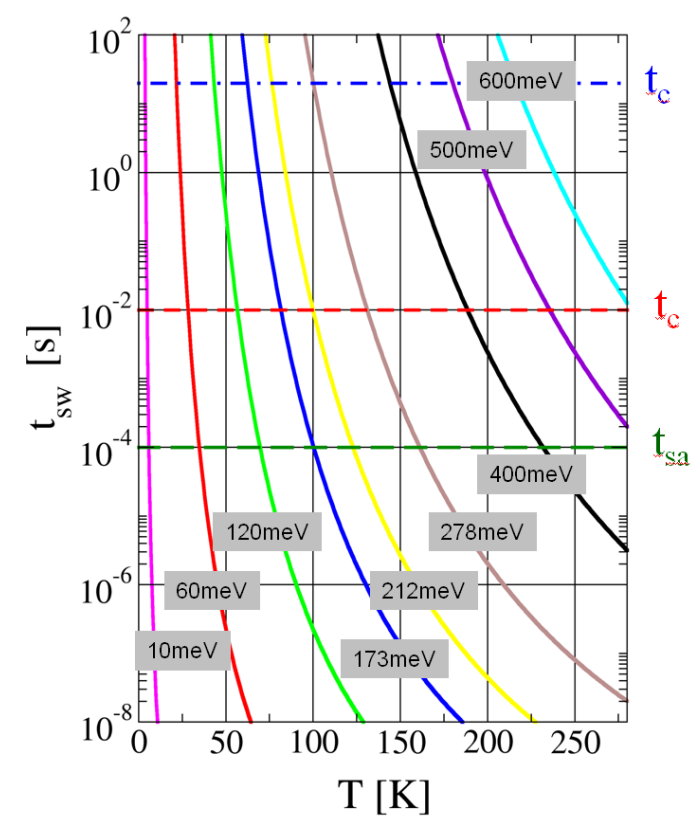

Figure 8. (color online) Switching time $t_{s w}$ as a function of temperature calculated for different energy barriers from 10 to $600 \mathrm{meV}$. The dashed line (red online) at $10^{-2} \mathrm{~s}$ and the dot dashed one (blue online) $20 \mathrm{~s}$ show the estimated coexistence time for the stretching speeds of $v=100 \mathrm{~nm} / \mathrm{s}$ and $v=0.05$ $\mathrm{nm} / \mathrm{s}$ used respectively in Fig. 1 and Fig. 3. The long dashed line (green online) show the sampling time of our experimental setup.

ii) The sampling time $t_{s a}$ of the acquisition setup, $10^{-4}$ $\mathrm{s}$ for our sampling frequency of $10 \mathrm{KHz}$.

iii) The "coexistence time"

$$
t_{c} \simeq \frac{\Delta z}{v}
$$

during which several metastable structures coexist. Here $v$ is the stretching speed of the electrodes and $\Delta z$ the elongation range where two metastable configurations exist. Assuming an order of magnitude of $1 \mathrm{~nm}$ (Figure 2) for $\Delta z$ we obtain a coexistence time of $t_{c} \simeq 10^{-2} \mathrm{~s}$ for $v=100 \mathrm{~nm} / \mathrm{s}$ (Figure 1) and $t_{c} \simeq 20 \mathrm{~s}$ for $v=0.05 \mathrm{~nm} / \mathrm{s}$ (Figure 3).

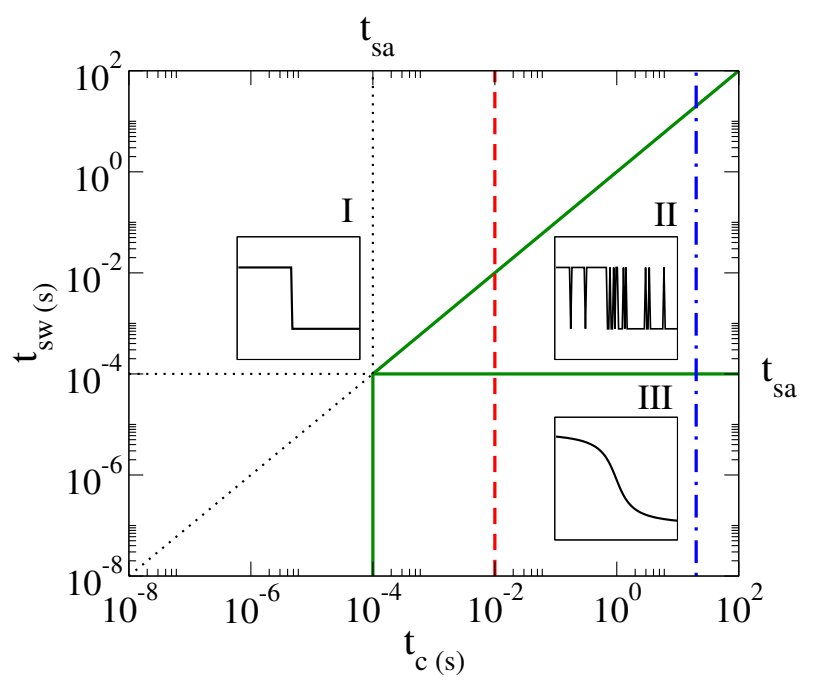

Figure 9. (color online) Switching time $t_{s w}$ versus coexistence time $t_{c}$ diagram showing the regions where the three cases discussed in the text take place. The sampling time of $10^{-4}$ $\mathrm{s}$ corresponds to our acquisition setup. The dashed line (red online) shows the estimated coexistence time $10^{-2} \mathrm{~s}$ for a stretching speed $v=100 \mathrm{~nm} / \mathrm{s}$ (Figure 1) and the dot dashed one (blue online) the coexistence time $20 \mathrm{~s}$ for $v=0.05 \mathrm{~nm} / \mathrm{s}$ (Figure 3). See text for more details.

Depending on the relative values of the above defined times one can observe different experimental behaviors as summarized in Fig. 9. This diagram shows the switching time versus the coexistence time. The diagonal line $t_{s w}=$ $t_{c}$ and the two $t_{c}=t_{s a}$ and $t_{s w}=t_{s a}$ lines allow to define six different regions where the three kinds of conductance traces can be observed.

I) In four of the regions, one can expect an abrupt conductance step in the $G(t)$ traces. These regions are the ones in Fig. 9 where the coexistence time $t_{c}$ is smaller than either the sampling $t_{s a}$ or the switching time $t_{s w}$.

$$
\begin{aligned}
t_{s a} & >t_{c}>t_{s w} \\
t_{s a} & >t_{s w}>t_{c} \\
t_{s w} & >t_{c}>t_{s a} \\
t_{s w} & >t_{s a}>t_{c}
\end{aligned}
$$

In these cases during the period of time corresponding to the coexistence, either the fluctuations cannot be observed due to experimental limitations or the system does not have time to fluctuate between the states. We thus observe only one switch, from the initial to the final state. 
II) Real time observation of switching events is possible only, when the switching time is framed by the sampling and coexistence times, i.e. :

$$
t_{c}>t_{s w}>t_{s a}
$$

This inequality can be re-written as a condition for the energy barrier :

$$
k \log \left(\nu_{0} t_{c}\right)>\frac{E_{b}}{T}>k \log \left(\nu_{0} t_{s a}\right)
$$

III) Finally, when the sampling time is larger than the switching time

$$
t_{c}>t_{s a}>t_{s w}
$$

the limited bandwidth of the acquisition setup does not allow the observation of the switching process, but only a mean value of the conductance relative to the time spent in every state during the stretching of the junction. It corresponds to a smooth transition from the conductance of the initial state to the conductance of the final state.

Under the experimental conditions of Fig. 1, sampling time $t_{s a}=10^{-4} \mathrm{~s}$ and an estimated coexistence time $t_{c} \simeq$ $10^{-2} \mathrm{~s}$, Fig. 9 shows that the three behaviors are possible but the temporal window for real time observation of the switching is very narrow. In the conductance traces we only observe abrupt (I) or smooth (III) transitions between two consecutive conductance plateaus. At the experimental temperature of 100K, Fig. 8 show that the switching can only be observed if the energies barriers are between 173 and $212 \mathrm{meV}$. From a statistical point of view, this condition are not likely to be fulfilled in most experiments.

The lower stretching speed of $0.05 \mathrm{~nm} / \mathrm{s}$ used in Fig. 3 results in a wider temporal window as can be seen in Fig. 9. At $100 \mathrm{~K}$, it corresponds to energy barriers between 173 and $278 \mathrm{meV}$. Temperatures about those of liquid hydrogen seems to optimize the observation of such traces. The range of energy barriers is reduced to $6<$ $E_{b}<10 \mathrm{meV}$ at $4 \mathrm{~K}$, and increased to $509<E_{b}<637$ $\mathrm{meV}$ at room temperature. It is probably the reason why experiments reported at these temperatures rarely show large fluctuation in the conductance traces.
Let us finally note that the $G(t)$ traces recorded in these conditions are more complex than a simple two level system. The histogram of the conductance of the trace, plotted in Fig. 3, shows that the system oscillates between, at least, four distinct states, associated to different conductances.

\section{CONCLUSION}

In this paper, we have reported an experimental and theoretical study of the transition between two consecutive conductance plateaus as observed during the breaking of gold atomic sized contacts at 100K. Our STM experiments performed at $100 \mathrm{~K}$ have shown that the transition between the plateaus can take place as an abrupt conductance step, a smooth quasicontinuous change or large amplitude conductance fluctuations. As has been reported before in the literature, the molecular dynamics simulations performed using a non-orthogonal tightbinding Hamiltonian have shown that during the stretching, the system evolves by elastic deformations followed by plastic rearrangements. A more detailed static study have shown that for a given deformation there are several structures having close and competing energies. The energy barriers between these structures are in the 0-500 meV range.

Finally, we have shown that depending on the relation between three characteristic times, the experimental conditions to observe the three different conductance traces can be explained. In order to observe the real time fluctuations related with the transitions between several structures using typical experimental setups liquid hydrogen temperatures (around 100K) seem to better correspond to the characteristic energy barriers.

\section{ACKNOWLEDGMENTS}

We acknowledge financial support from the French program PNANO-2008 "SUD - Spintronique à Une Dimension" under the project (ANR-08-NANO-P056-36).

\section{REFERENCES}

${ }^{4}$ L. Olesen, E. Laegsgaard, I. Stensgaard, F. Besenbacher, J. Schiøtz, P. Stoltze, K. W. Jacobsen, and J. K. Nørskov, Phys. Rev. Lett. 72, 2251 (1994).

5 J. M. Krans, C. J. Muller, N. van der Post, F. R. Postma, A. P. Sutton, T. N. Todorov, and J. M. van Ruitenbeek, Phys. Rev. Lett. 74, 2146 (1995).

6 L. Olesen, E. Laegsgaard, I. Stensgaard, F. Besenbacher, J. Schiøtz, P. Stoltze, K. W. Jacobsen, and J. K. Nørskov, Phys. Rev. Lett. 74, 2147 (1995). 
7 Z. Gai, Y. He, H. Yu, and W. S. Yang, Phys. Rev. B 53, 1042 (1996).

8 J. N. Armstrong, R. M. Schaub, S. Z. Hua, and H. D. Chopra, Phys. Rev. B 82, 195416 (2010).

9 T. Leoni, R. Zoubkoff, S. Homri, N. Candoni, P. Vidakovic, A. Ranguis, H. Klein, A. Saul, and P. Dumas, Nanotechnology 19, 355401 (2008).

10 H. E. van den Brom, A. I. Yanson, and J. van Ruitenbeek, Physica B: Condensed Matter 252, 69 (1998).

11 M. Tsutsui, S. Kurokawa, and A. Sakai, Appl. Phys. Lett. 90, 133121 (2007).

12 M. Huntington, J. Armstrong, M. Sullivan, S. Hua, and H. Chopra, Phys. Rev. B 78, 035442 (2008).

13 H. Klein, W. Blanc, R. Pierrisnard, C. Fauquet, and P. Dumas, Eur. Phys. J. B 14, 371 (2000).

14 T. Leoni, M. Alwan, H. Klein, N. Candoni, A. L. Lereu, and P. Dumas, Global Journal of Physical Chemistry 2, 97 (2011).

15 A. Marchenkov, Z. Dai, C. Zhang, R. Barnett, and U. Landman, Phys. Rev. Lett. 98, 046802 (2007).

16 R. E. Cohen, M. J. Mehl, and D. A. Papaconstantopoulos, Phys. Rev. B 14, 694 (1994).

17 M.J.Mehl and D. A. Papaconstantopoulos, Phys. Rev. B
54, 4519 (1996)

18 M. Elstner, D. Porezag, G. Jungnickel, J. Elsner, M. Haugk, T. Frauenheim, S. Suhai, and G. Seifert, Phys. Rev. B 58, 7260 (1998).

19 V. L., Phys. Rev. B 159, 98 (1967).

${ }^{20}$ H. J. C. Berendsen, J. P. Postma, W. F. van Gunsteren, A. Dinola, , and J. R. Haak, J. Chem. Phys. 81, 3684 (1984).

21 Y. Kondo and K. Takayanagi, Science 289, 606 (2000).

22 E. Tosatti, S. Prestipino, S. Kostlmeier, A. D. Coraso, and F. D. D. Tolla, Science 291, 288 (2001).

${ }^{23}$ R. Zoubkoff and A. Saúl, to be published.

24 D. Sanchez-Portal, E. Artacho, J. Junquera, P. Ordejon, A. García, and J. M. Soler, Phys. Rev. Lett. 83, 3884 (1999).

25 B. Legrand, G. Treglia, M. C. Desjonqueres, and D. Spanjaard, J. of Phys. C: Solid State Physics 19, 4463 (1986).

${ }^{26}$ G. Henkelman, B. Uberuaga, and H. Jonsson, J. Chem. Phys. 113, 9901 (2000).

27 G. Henkelman and H. Jonsson, J. Chem. Phys. 113, 9978 (2000).

28 J. Lynn, H. Smith, and R. Nicklow, Phys. Rev. B 8, 3493 (1973). 